\title{
1 Keystone species determine the productivity of synthetic microbial
}

\section{2 biofilm communities}

3 Xinli Sun ${ }^{1,2}$, Jiyu Xie ${ }^{1}$, Daoyue Zheng ${ }^{1}$, Wei Wang ${ }^{1}$, Riyan Xia ${ }^{1}$, Ruifu Zhang ${ }^{1}$, Ákos T. Kovács ${ }^{2 *}$,

$4 \quad$ Zhihui $\mathrm{Xu}^{1^{*}}$, Qirong Shen ${ }^{1}$

51 Jiangsu Provincial Key Lab of Solid Organic Waste Utilization, Jiangsu Collaborative Innovation

6 Center of Solid Organic Wastes, Educational Ministry Engineering Center of Resource-Saving

7 Fertilizers, The Key Laboratory of Plant Immunity, Nanjing Agricultural University, 210095 Nanjing,

8 Jiangsu, Peoples R China

92 Bacterial Interactions and Evolution Group, DTU Bioengineering, Technical University of Denmark,

102800 Kongens Lyngby, Denmark

11 Xinli Sun and Jiyu Xie contributed equally to this work.

$12{ }^{*}$ Corresponding authors: Zhihui Xu and Ákos T. Kovács

13 E-mail: xzh2068@njau.edu.cn, atkovacs@dtu.dk

15 Abstract

16 Microbes typically reside in multi-species communities, whose interactions have considerable impacts

17 on the robustness and functionality of such communities. To manage microbial communities, it is

18 essential to understand the factors driving their assemblage and maintenance. Even though the

19 community composition could be easily assessed, interspecies interactions during community

20 establishment remain poorly understood. Here, we combined co-occurrence network analysis with

21 quantitative PCR to examine the importance of each species within synthetic communities (SynComs)

22 of pellicle biofilms. Genome-scale metabolic models and in vivo experiments indicated that the

23 biomass of SynComs was primarily affected by keystone species that are acting either as metabolic 
24 facilitators or as competitors. Our study suggests that a combination of co-occurrence network

25 analysis and metabolic modeling could explain the importance of keystone species in SynComs.

\section{Introduction}

27 In natural microbiome systems, hundreds or thousands of species occupy and interact with each other,

28 as well as the environment around them. To deal with this complexity, we can utilize synthetic communities (SynComs) to identify common principles governing and structuring microbiomes (Bengtsson-Palme, 2020). Several SynComs with moderate complexity and high controllability have been developed to represent different natural environments (Blasche et al., 2017; Chan et al., 2017; Fu et al., 2020; Lebeis et al., 2015; Weiss et al., 2021). Such knowledge is essential to disentangle complex community interactions and to provide powerful tools for biotechnological, medical, or agricultural purposes (Abram, 2015; Cavaliere et al., 2017; Cho and Blaser, 2012; Fitzpatrick et al., 2020; Gómez-Godínez et al., 2021). To harness this potential, it is essential to preserve both the microenvironments in which these beneficial processes can take place, as well as the microbial populations that contribute to these processes. Based on the study of SynComs, researchers have found that the assemblage and robustness of communities can be affected by several factors including pH (Ortiz et al., 2021), spatial distribution (Liu et al., 2019), initial abundance (Gao et al., 2020, 2019), niche specificity (Estrela et al., 2021), nutrient availability (Ratzke et al., 2020), keystone species (Niu et al., 2017) and so on. Nevertheless, many questions remain about how diversity is maintained, and ultimately, how the community function can be preserved and controlled.

Multiple species are widely distributed in nature as surface-associated communities also referred to as biofilms (Sadiq et al., 2021). The biofilm formation process consists of the planktonic stage, initial attachment to a surface, the building of micro-aggregates, biofilm maturation, and biofilm dispersal (Lee and Yoon, 2017). These multi-species biofilm communities assemble as a result of interspecies interactions, which determine not only the composition and distribution but also their biological function. Compared with solitary bacteria, species residing in multispecies biofilms can gain fitness advantages, including enhanced biomass production (Ren et al., 2015), stress tolerance (Lee et al., 
51 et al., 2000) through cooperative interactions. In contrast, ecological competition is more pervasive in

52 multispecies biofilms (Foster and Bell, 2012; Oliveira et al., 2015). Bacteria actively inhibit competitors

53 by producing toxins, biosurfactants, and antimicrobials (Rendueles and Ghigo, 2012), or indirectly

54 impair the fitness of neighbors through nutrient scavenging. The spatial structuring property of biofilms

55 supports the intermixing of cooperating species and the partitioning of niches by competing species.

56 Understanding how these interactions affect the formation of multi-species biofilms is therefore

57 important for their utilization or inhibition.

58 Despite a wealth of information on microbiota composition gathered from sequencing techniques, the

59 knowledge of microbial interactions that occur in the microbiota is severely lacking. Building a co-

60 occurrence network from high-throughput sequence data is a common method of deriving hypotheses

61 from these massive data (Faust et al., 2012). For instance, it has been applied to link taxa to biological

62 functions of interest (Wei et al., 2019), to identify potential biotic interactions (Durán et al., 2018), and

63 to explore habitat differentiation (Barberán et al., 2012). Another emerging method for exploring

64 microbial interactions is genome-scale metabolic modeling, which can provide insights into metabolic

65 interaction potential and metabolic resource overlap in multi-species communities (Zelezniak et al.,

66 2015; Zorrilla et al., 2021). Microbiome network analysis and metabolic modeling could guide the

67 study of SynComs for animal and plant health (Heinken et al., 2021; Poudel et al., 2016). However,

68 the ecological relevance of predicted interactions remains poorly understood (Faust, 2021). The

69 connectedness and strength of positive or negative interactions are not experimentally verified. The

70 main challenge of studying microbial interactions in SynComs is fast and accurate quantification of

71 the abundance of each species. This can be achieved by $16 \mathrm{~S}$ amplicon sequencing, colony-forming

72 unit counting, fluorescent labeling, and quantitative PCR. Both of the first two approaches are simple

73 to employ, but they can be time-consuming. While the first two approaches are more convenient but

74 time-consuming, the latter two approaches are more efficient but require extensive initial time and

75 effort.

76 In this study, we constructed SynComs using isolates from a rhizosphere and predicted their

77 interaction by analyzing co-occurrence networks. The network analysis was validated by quantitative 
assays. The potential metabolic interactions were further investigated through experiments and metabolic modeling. The results suggested that keystone species determine community productivity through metabolic exchanges or resource competition. We propose that our study could inform the

81 rational design of synthetic communities.

\section{Results}

83 C. rhizoplanae and $P$. eucrina were predicted to be keystone species in the eleven-species

\section{SynCom}

85 In a previous study, we screened for cooperative bacteria for the plant-growth-promoting bacteria 86 Bacillus velezensis SQR9, by studying the effect of strain SQR9 on rhizosphere microbiome 87 composition (Sun et al., 2021). Parallel to those experiments, we collected and co-cultured the 88 rhizosphere soil with strain SQR9 in a liquid medium to form pellicle biofilms (Figure 1-figure 89 supplement $1 \mathrm{~A})$. Using an amplicon sequencing method, we determined the bacterial composition of 90 the corresponding biofilm and the solution underneath. Consequently, 15 genera and 2 families were 91 predicted to co-exist in the culture condition (Figure 1-figure supplement 1B). From our bacterial 92 collection, we selected 11 isolates that corresponded with the predicted co-existing taxa and 93 combined them into a synthetic biofilm community (Figure 1-figure supplement 2). These isolates 94 are derived from three different phyla: Firmicutes, Proteobacteria, and Bacteroidetes.

A

\section{5}

Collect rhizosphere soil Biofilm co-culture with

B. velezensis SQR9

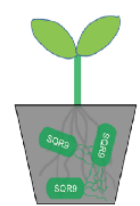

Black soil

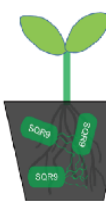

Paddy soil
Biofilm Solution 
bioRxiv preprint doi: https://doi.org/10.1101/2022.01.23.477386; this version posted February 12, 2022. The copyright holder for this preprint (which was not certified by peer review) is the author/funder, who has granted bioRxiv a license to display the preprint in perpetuity. It is made available under aCC-BY-NC-ND 4.0 International license.

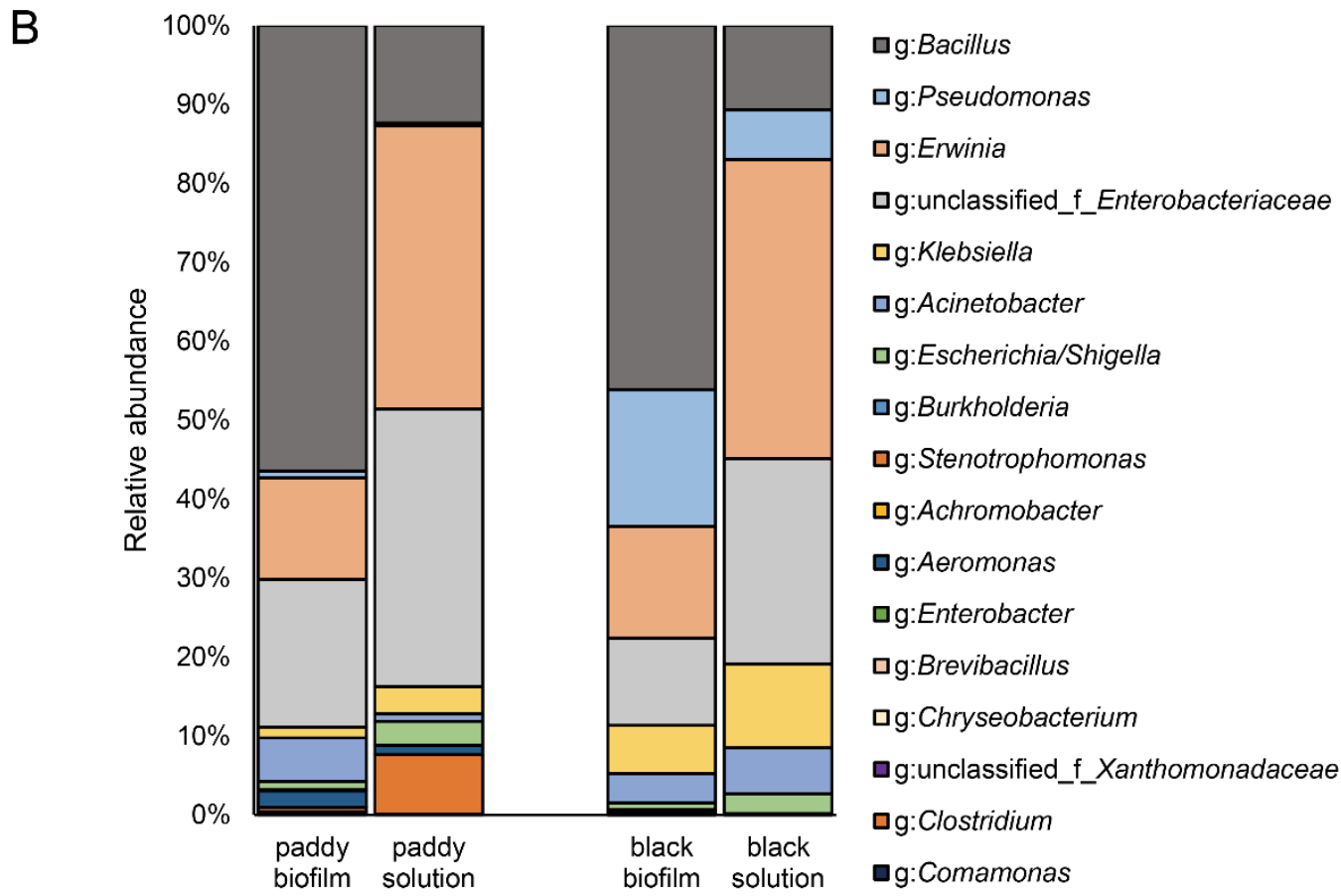

Figure 1-figure supplement 1. Origin of the initial 11 isolates. (A) Schematic diagram of the experimental setup. Rhizospheric black soil and paddy soil were collected from cucumber plants. The soil microbiota were co-cultivated with $B$. velezensis SQR9 at $30^{\circ} \mathrm{C}$ in TSB medium to form pellicle biofilms. After 24 hours of cultivation, the pellicle and the solution underneath were collected separately $(n=3)$. The samples were sent for 16S rDNA amplicon sequencing. (B) Microbiome composition of the biofilm and solution. Based on OTU clustering and the RDP database taxonomic classification, 15 genera and 2 families were identified. Eleven matching isolates were selected from our laboratory bacteria collection (Sun et al., 2021).

\section{7 isolates}

isolated from the rhizosphere of cucumber plant (Sun et al., 2021)

\section{6 isolates}

selected for the reduced SynComs and genome

sequencing based on the network analyses 
101 We evaluated the stability of this SynCom by observing the compositional change of individual

102 members using 16S rRNA gene amplicon sequencing. Several time points were selected to represent

103 the development of a biofilm: initial aggregation (2d), establishment (4d), maturation (6d), and

104 dispersal (8d). Chr (Chryseobacterium rhizoplanae) was identified to be the predominant species

105 throughout the biofilm development (Figure 1A). Three species Ach (Achromobacter denitrificans),

106 Pxa (Pseudoxanthomonas japonensis), and Ste (Stenotrophomonas maltophilia) rapidly declined and

107 could not establish themselves in the community. Network co-occurrence analysis was employed to

108 examine the correlations among the species during succession. The correlations among the species

109 varied from less to more (Figure 1B). Pan (Pantoea eucrina) was consistently positively correlated

110 with other species, while Chr was consistently negatively correlated with other species. These two

111 species were predicted to act as hub taxa in this multi-species consortium. In the subsequent

112 experiments, we examined the interactions of six of the most abundant species, including Pan, Chr,

113 Com (Comamonas odontotermitis), Aci (Acinetobacter baumannii), Ent (Enterobacter bugandensis),

114 and Pse (Pseudomonas stutzeri). Other species were excluded either due to their low abundance at

115 the biofilm maturation stage, or due to the lack of correlation with the other species.

A

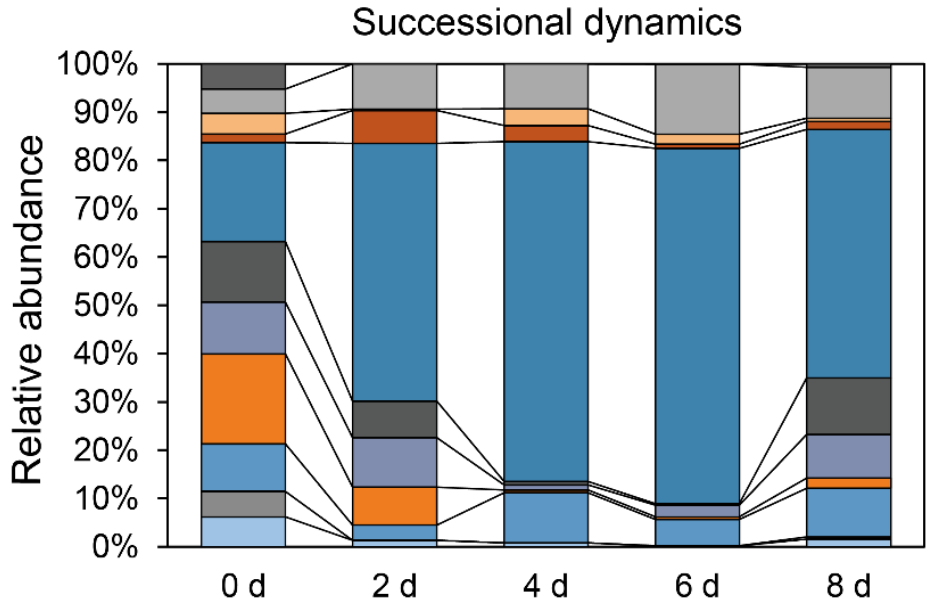

Species

$\square$ Achromobacter denitrificans XL100

$\square$ Acinetobacter baumannii XL380

$\square$ Bacillus velezensis SQR9

- Burkholderia contaminans XL73

口 Chryseobacterium rhizoplanae XL97

- Comamonas odontotermitis WLL

$\square$ Enterobacter bugandensis XL95

口 Pantoea eucrina XL123

$\square$ Pseudomonas stutzeri XL272

$\square$ Pseudoxanthomonas japonensis XL7

$\square$ Stenotrophomonas maltophilia XL133 
B

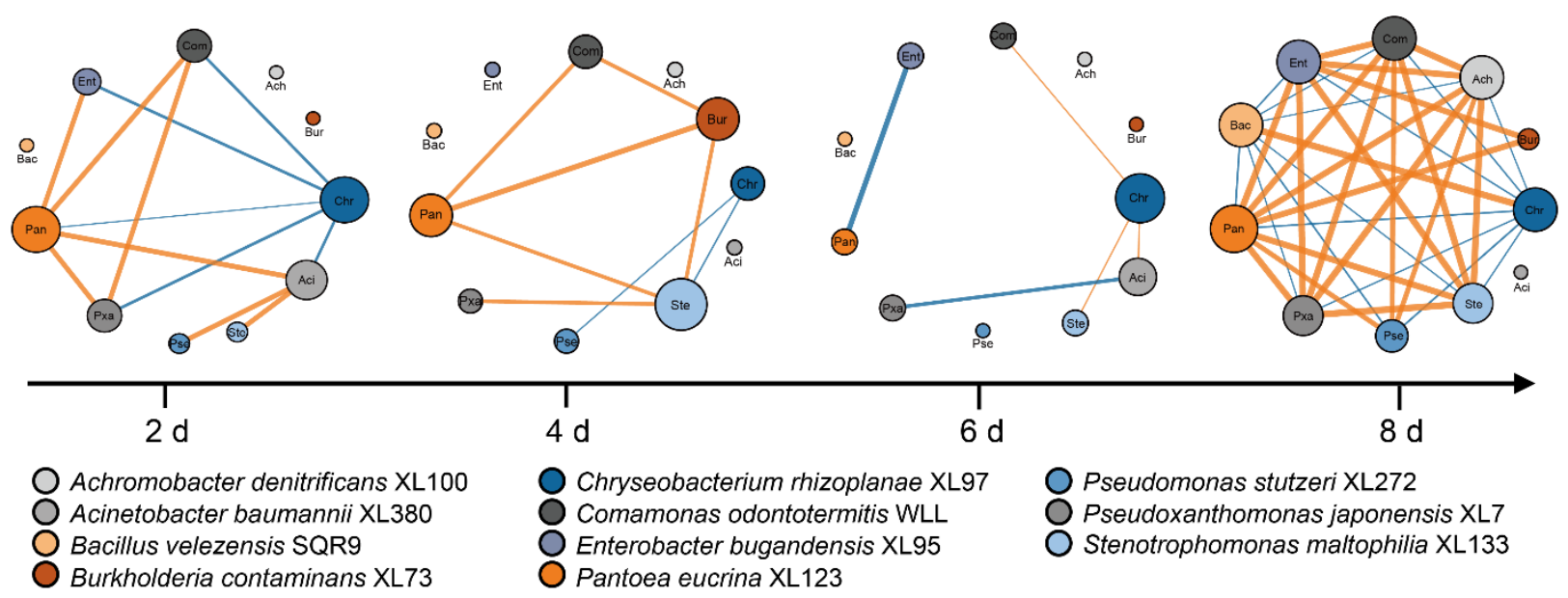

Figure 1. Dynamic changes of the initial 11-species consortium composition in the biofilm. (A) The relative abundance of isolates determined by $16 S$ rRNA gene amplicon sequencing method $(n=8)$. (B) Co-occurrence of bacterial clades within biofilm communities. Each node represents a bacterial species, node size is proportional to betweenness centrality. Line width indicates the interactive strength of interaction, and color indicates the sign of association (orange indicates positive, blue indicates negative).

Figure supplement 1 . Origin of the initial 11 isolates.

Figure supplement 2. A flow chart of isolates used for the study, their origin and selection criteria.

\section{Productivity of multi-species biofilm was affected by key members}

To test the central role that Pan and Chr play in biofilm development, we evaluated the relative productivity of different combinations of the six strains. "Add-in" and "Removal" strategies were applied (Figure 2A). According to the "Add-in" strategy, the "Basic community" consisted of four members (Com, Aci, Ent, and Pse), upon which we added the predicted positive or negative member to the community and compared the total biomass. Based on our hypothesis, we anticipated that supplementation of the positive member would increase total biomass, while we anticipated the opposite for the negative member. On the other hand, the "Removal" strategy included all the six species as a full community, then one isolate was dropped out to obtain the five-species reduced communities. Biofilm productivity was assessed in two ways: fresh weight and total cell numbers

131 (Figure 2A).

132 Compared to the "Full community", "Rm Chr" and "Rm Com" resulted in a higher biofilm weight, 133 whereas "Rm Ent" and "Rm Pan" resulted in a lower biofilm weight (Figure 2B). This indicated that 134 these four species are important to maintain the productivity of this synthetic community. However, 135 the contribution of each species to the population productivity could not be determined using this approach. The previously used amplicon sequencing method was relatively slow and could only 
137 provide information on the relative abundance. The main challenge in determining the composition is

138 to establish a fast and accurate method for quantifying the absolute abundance of each species. To

139 achieve this goal, we performed a genome-scale comparison and identified strain-specific genes in

140 each species. We designed multiple primers to amplify these genes and tested their specificity against

141 other species. Consequently, strain-specific primers were developed to selectively amplify only one

142 isolate from the six-members community (Table 1). Using these primers, we were able to determine

143 the contribution of each isolate to the community productivity. "Rm Chr" reached the highest number

144 of total cell numbers, while "Rm Pan" greatly decreased the total cell numbers (Figure 2C). Taken

145 together, we identified Pan and Chr as the keystone positive and negative species in terms of 146 community productivity, respectively.

A

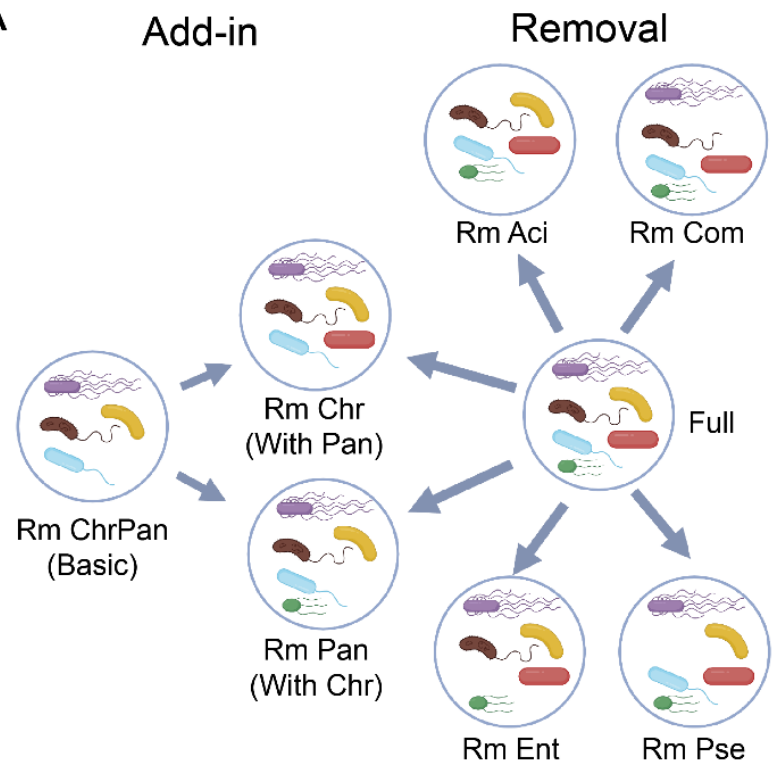

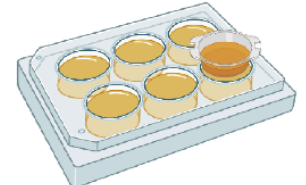

Biofilm communities

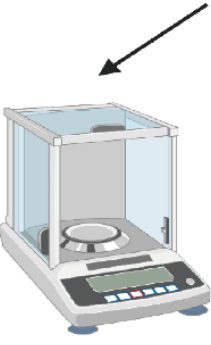

Weight
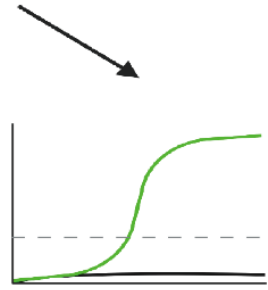

Cell numbers quantification by qPCR 

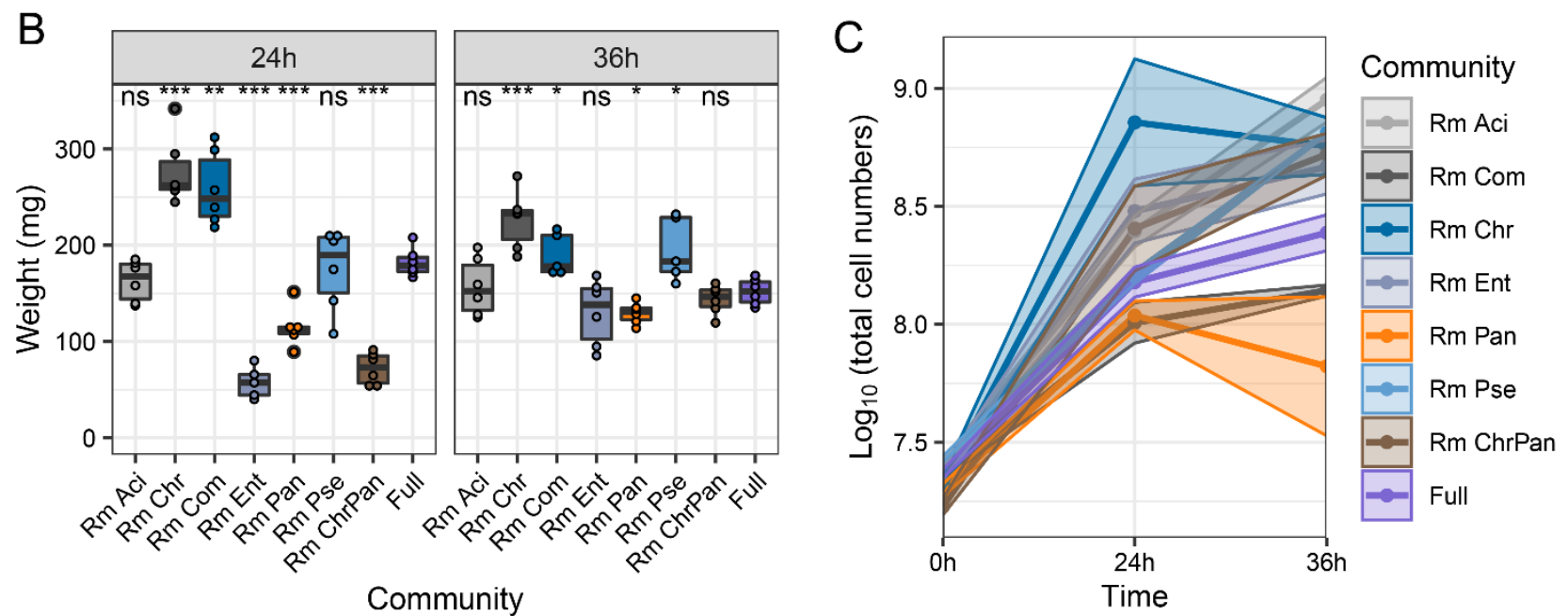

Fig 2 Productivity of the reduced SynComs. (A) Schematic diagram of experimental setup. Add in: positive or negative species were added in the four-species community (Rm ChrPan) to create five-species communities. Removal: each isolate was dropped out from the six-species community (Full) to create five-species communities. Biofilm biomass were quantified by fresh weight and cell numbers were quantified by qPCR. Diagrams were generated in Biorender. (B) Biofilm biomass quantified by fresh weight ( $n=5-6)$. The "Full community" was set as the control group. Asterisks indicate statistically significant according to unpaired student's $t$ test via $\mathrm{R}\left({ }^{* * *} p<0.001,{ }^{* *} p<0.01\right.$, ${ }^{*} p<0.05$ ). (C) Total cell numbers in the communities $(n=6)$. Color strip indicates standard deviation. All represents the full six-species communities, Rm Aci, Rm Chr, Rm Com, Rm Ent, Rm Pan, Rm Pse represent the five-species communities resulting from the removal of Acinetobacter baumannii XL380, Chryseobacterium rhizoplanae XL97, Comamonas odontotermitis WLL, Enterobacter bugandensis XL95, Pantoea eucrina XL123 and Pseudomonas stutzeri XL272, respectively. Rm ChrPan represents the four-species community resulting from the removal of $C$. rhizoplanae and $P$. eucrina.

\section{community}

151 The compositions of different communities were clustered based on the Bray-Curtis dissimilarity index

152 (Figure 3A-C). After 24 hours, the communities clustered into two main groups: "Full community", 153 "Basic community" (Rm ChrPan), "Rm Pan", and "Rm Chr" communities clustered together, whereas 154 the other communities were grouped in one branch. The upper branch was dominated by Ent, the lower branch by Pan. Based on the hierarchical clustering, Pan or Chr played deterministic roles in species composition by influencing the relative abundance of Ent. Additionally, the random forest analysis revealed that Pan played a major role in determining the differences of community compositions (Figure 3D). After 36 hours, the communities were divided into two branches without any apparent disciplinarian. We found that the proportion of Pan cells was highest in the "Rm Ent" community, while the proportion of Ent cells was highest in the "Rm Pan" community (Figure 3C). 
A on

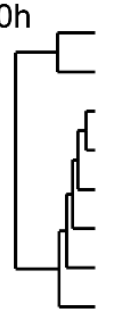

$$
\begin{array}{r}
\text { Rm Pan } \\
\text { Rm ChrPan } \\
\text { Rm Pse } \\
\text { Rm Aci } \\
\text { Full } \\
\text { Rm Ent } \\
\text { Rm Com } \\
\text { Rm Chr }
\end{array}
$$

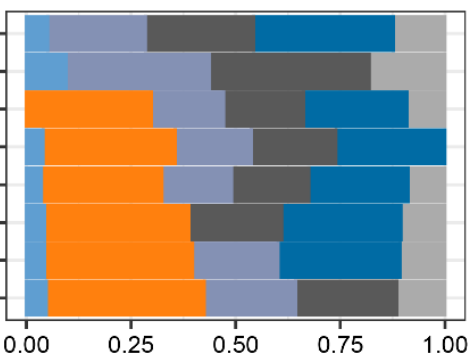

Relative abundance
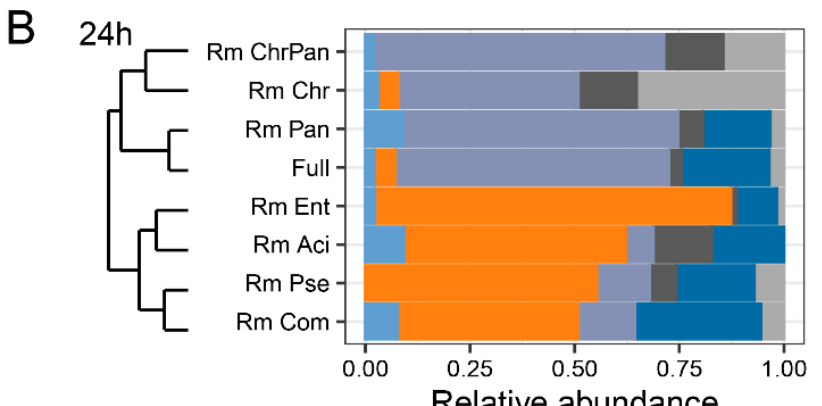

C 36h
Relative abundance

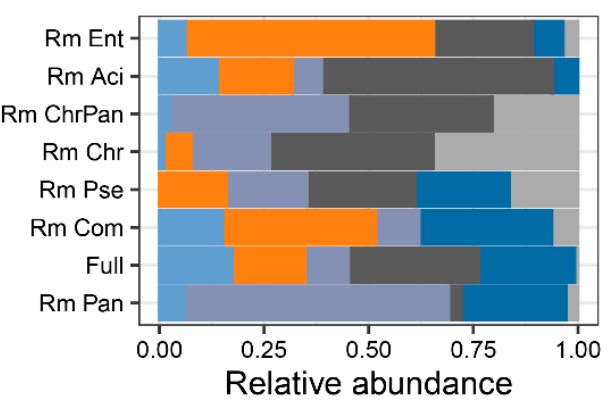

Species

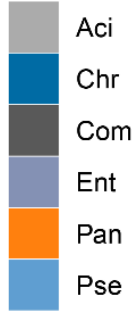

D

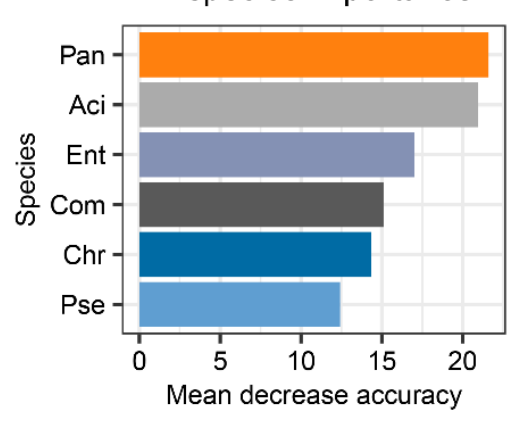

$\mathrm{E}$

36h species importance

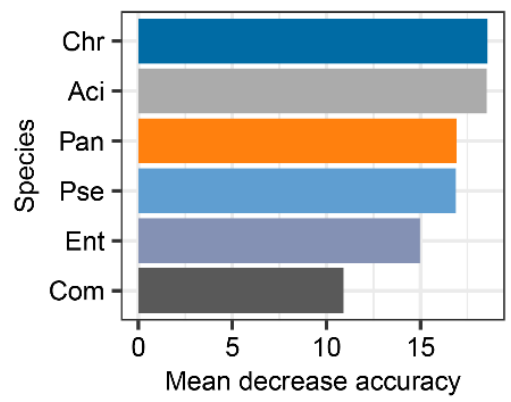

FIG 3 Species composition and importance. (A-C) Hierarchical clustering analysis of the synthetic community composition $(n=6)$. Samples include a six-species communities (Full), a four-species community ( $\mathrm{Rm} \mathrm{ChrPan}$ ) and six five-species communities (Rm communities). Rm Aci, Rm Chr, Rm Com, Rm Ent, Rm Pan, Rm Pse represent the five-species communities resulting from the removal of Acinetobacter baumannii XL380, Chryseobacterium rhizoplanae XL97, Comamonas odontotermitis WLL, Enterobacter ludwigii XL95, Pantoea eucrina XL123 and Pseudomonas stutzeri XL272, respectively. The samples were collected at the initial inoculation (A), 24h (B) and 36h (C). (D-E)

Species importance in the SynComs as predicted by the random forest analysis.

Metabolic facilitation explained the positive interaction

To gain insights into the metabolic interaction potential of the SynCom members, we reconstructed genome-scale metabolic models, using the M9 glucose minimal medium as the input medium. We derived the likely exchanged metabolites across communities and the strength of metabolic coupling (Figure 4A-D). Pan was the principal donor in the communities with Pan, whereas Ent was the principal donor in the communities without Pan. This result explained the dominance of these two species in the SynComs. In contrast, adding Chr to the SynComs had little effect on the global 
173 metabolic exchanges. Chr acted more as a receiver rather than a donor in the "Full community"

174 (Figure 4A). In addition, the add-in or removal of keystone species also affected the proportion of 175 exchanged compounds (Figure 4E-H). Amino acids and phosphates were the major categories of 176 metabolites exchanged across communities. When the positive member Pan was removed, we 177 observed higher reliance on amino acid cross-feeding and lower dependence on organoheterocyclic 178 compounds exchange. Moreover, we highlighted the interactions involving Pan as the donor in the 179 "Full community" (Figure 4I) and in the "Add Pan" (Rm Chr) community (Figure 4J). We observed that 180 the metabolic contribution of Pan benefited all community members.

181 Growth assays were used to determine the accuracy of the metabolic models. Firstly, all species 182 except Chr were able to grow individually in the M9 glucose minimal medium (data not shown), thus 183 five of them have the potential to serve as donors. Secondly, growth in the spent culture medium was 184 utilized to determine metabolic interaction potential. Each isolate was cultured in the M9 glucose 185 medium until the glucose was undetectable. We obtained sterile spent medium and used it to cultivate 186 every other member of the community and themselves. The maximum growing capacity was 187 determined and defined as growth facilitation (Figure 4K). Results showed that the spent medium 188 from Pan and Ent could support the growth of all other isolates. Pse's metabolic by-products were 189 also capable of supporting the growth of three isolates. Aci, Com, and Chr acted only as receivers but 190 not donors. These results fully supported the metabolic models, suggesting Pan and Ent contributed 191 to the overall productivity by cross-feeding, whereas Chr acted as a cheater. 


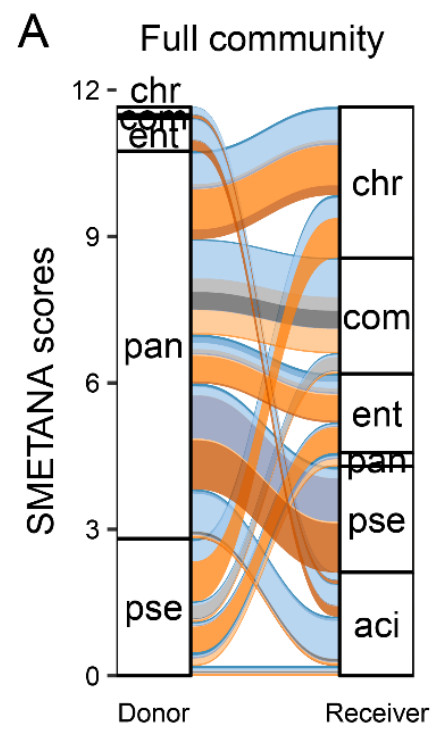

\section{B Add Pan (Rm Chr)}

12 -
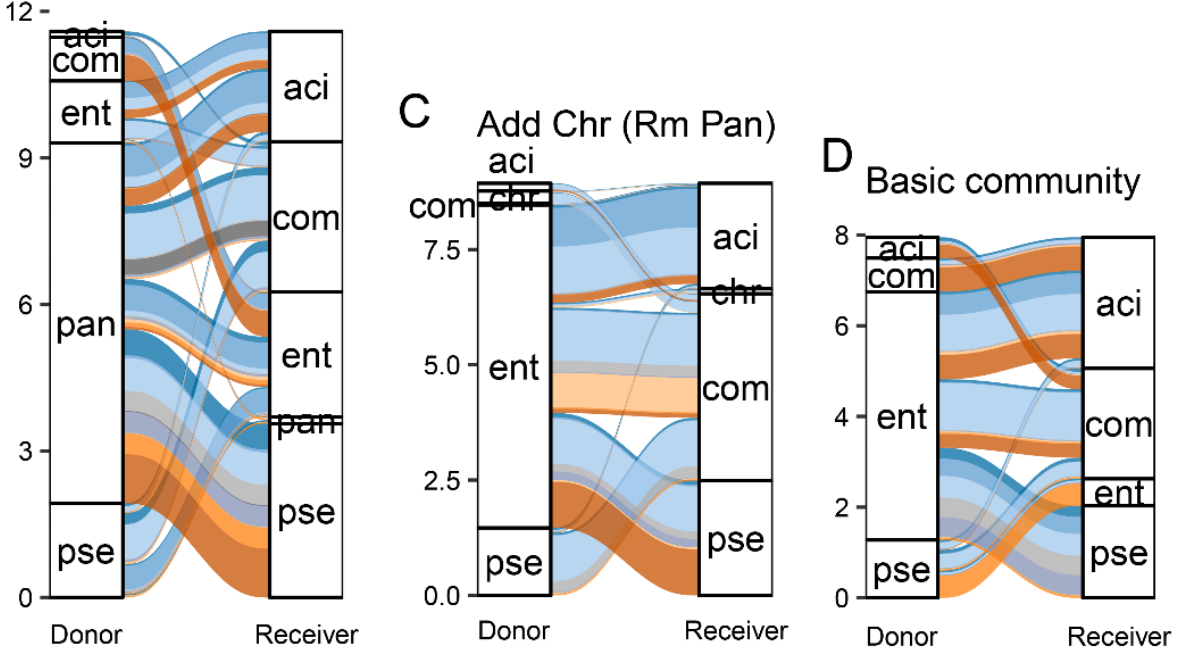

E Full community

F Add Pan (Rm Chr)

G Add Chr (Rm Pan)

$\mathrm{H}$ Basic community
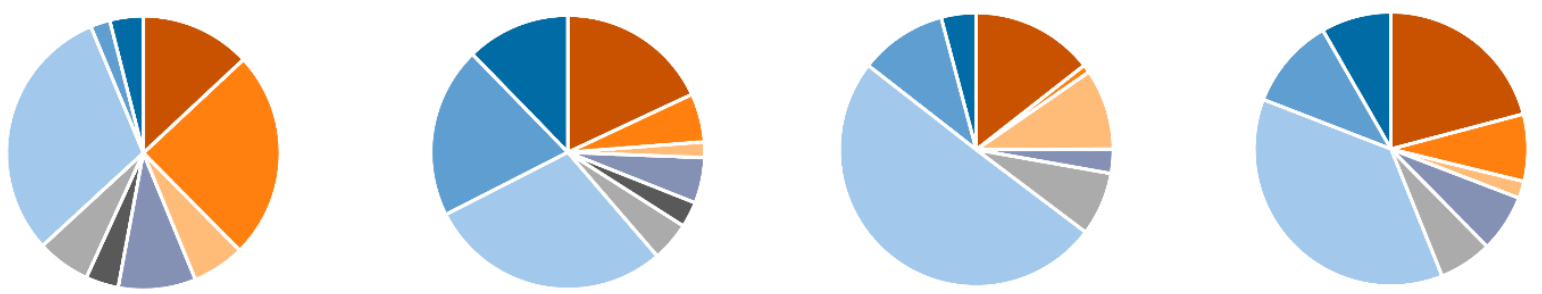

I Full community

J Add Pan (Rm Chr)

K

Growth facilitation
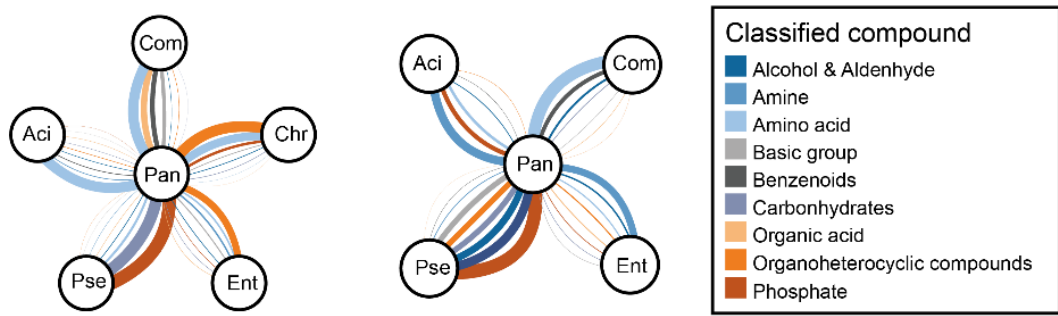

$\left(\mathrm{OD}_{600}\right)$

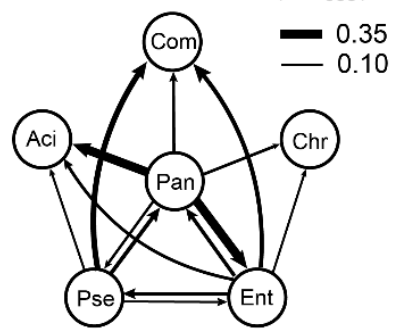

Fig 4 Metabolic facilitation. (A-J) Metabolic interaction potential simulated by genome-scale metabolic modelling. (A-D) Alluvial diagrams showing compounds exchanged within communities. Thickness of lines are SMETANA scores. (E-H) Pie charts showing the proportion of compounds exchanged within communities. (I-J) Flower plots showing interactions involving positive species $P$. eucrina XL123 (centered in each panel) as a donor across communities. Thickness of lines are proportional to magnitude of SMETANA score. Colors of all plots are the classification of compounds. (K) Growth facilitation assessed by growth in spent medium. The spent medium was prepared by growing the corresponding species in M9 medium with $1 \%$ glucose till the glucose was under detection then filter sterilized. The species were grown in the filtered spent medium for $24 \mathrm{~h}$ and the growth were measured as the $\mathrm{OD}_{600}$. Line width indicates the $O \mathrm{D}_{600}$.

\section{Resource competition explained the negative interaction}

194 Higher-order interactions may further be explained by metabolic competition. Using metabolic modeling, the metabolic resource overlap was simulated (Figure 5A). We observed high metabolic resource overlap across all communities, indicating intense resource competition among SynCom 
possibility of direct competition, we performed spot-on-lawn and pair-wise spot assays on TSB agar plates (Figure 5B-C). Both Aci and Ent completely inhibited the growth of Com. Similarly, Chr and Ent

201 inhibited Pse growth. However, these inhibitions were not caused by direct antagonism as no inhibition zones were observed in the respective spot-on-lawn assay plates (Figure 5B, red square). In light of these results, no clear boundary was observed between two strains when they were spotted adjacent to one another (Figure 5C). Interestingly, Pan and Ent could change the colony color of Chr from light yellow to white (Figure 5B, yellow square). We also assessed the carbon source metabolic ability by high-throughput phenotypic microarrays (Figure 5D). Ent and Pan were generalists that can utilize a wide variety of carbon sources. Other community members were specialized in using amino acids and organic acids as carbon sources while having a limited ability to utilize nucleotides, sugar alcohols, and sugars. Collectively, resource competition was widespread among the SynCom members.

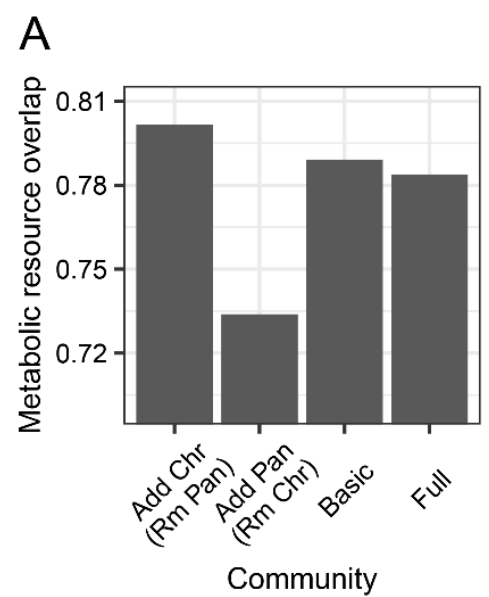

B

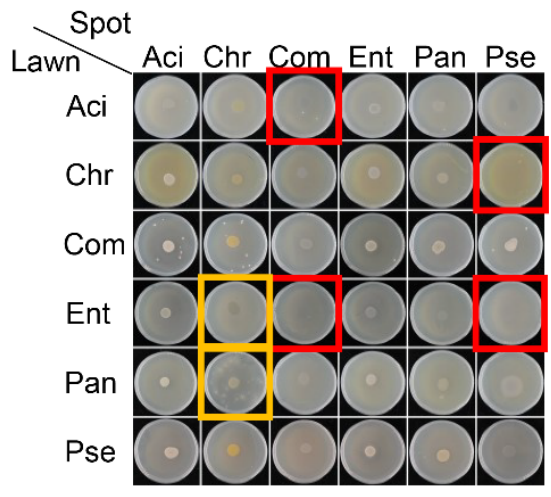

C<smiles>C1CCCCC1</smiles>
Left Aci Chr Com Ent Pan Pse

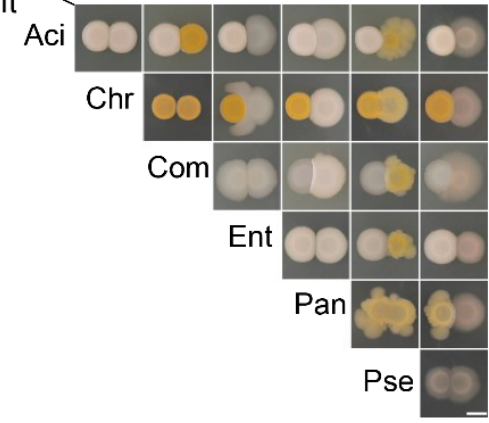

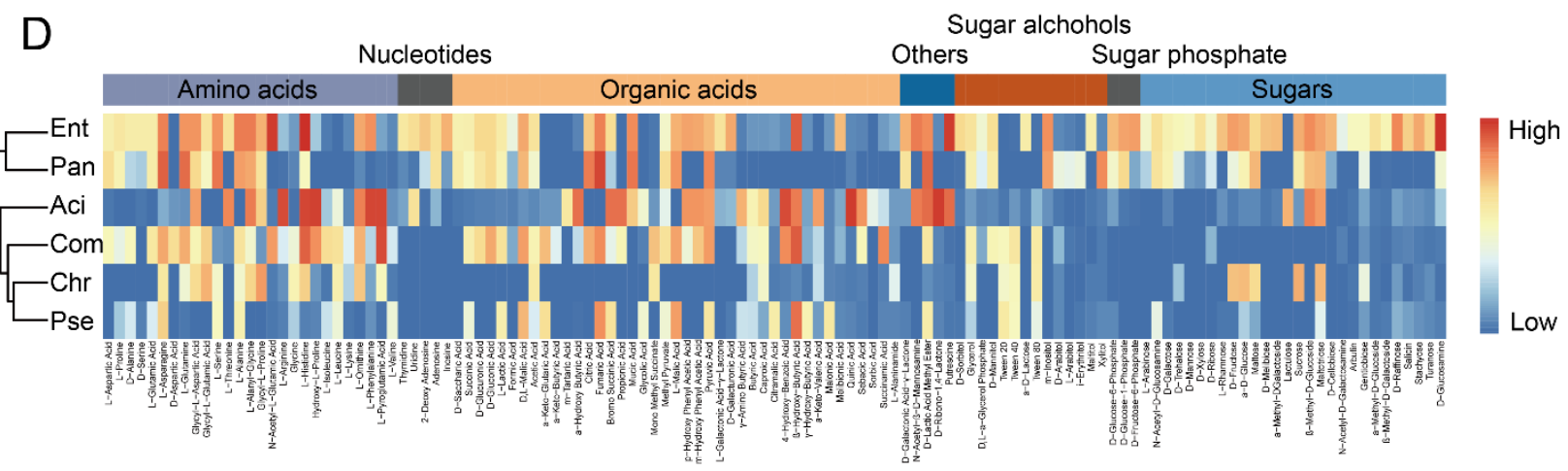


Fig 5 Resource competition. (A) Bar chart showing the metabolic resource overlap simulated by the metabolic modeling. (B) Spot-on-lawn assays. The lawn species were spread on TSB agar plates at an $\mathrm{OD}_{600}$ of 0.02 and dried, $5 \mu \mathrm{l}$ of the spot species were spotted on the center at an $\mathrm{OD}_{600}$ of 0.4 . The plate diameter is $6 \mathrm{~cm}$. Photos were taken after $48 \mathrm{~h}$ incubation at $30^{\circ} \mathrm{C}$. (C) Pair-wise spot assays. $5 \mu \mathrm{l}$ of the two species were plot next to each other at an $\mathrm{OD}_{600}$ of 1 . Scale bar represents $3 \mathrm{~mm}$. Photos were taken after $48 \mathrm{~h}$ incubation at $30^{\circ} \mathrm{C}$. (D) Carbon source metabolic ability measured by phenotype microarrays.

\section{Discussion}

214 A key concern in SynCom research is to understand how microbial interactions affect community 215 composition and productivity. In our study, we evaluated the contribution of each individual to 216 community productivity and revealed the metabolic interactions. Our SynCom system identified Pan

217 (P. eucrina) and $\operatorname{Chr}($ C. rhizoplanae) as important drivers of community interaction networks through 218 metabolic cross-feeding and resource competition.

219 Manipulating microbial consortia has a variety of applications; however, attempts to engineer 220 microbial consortia often fail to achieve the expected results owing to the unexpected effects of 221 interactions within the community. Although the practical application of this SynCom is not yet clear, 222 accurate prediction of the species composition is the first step toward manipulating microbial consortia 223 (Widder et al., 2016). In this study, we assessed the SynCom composition using the amplicon sequencing method and qPCR. In the initial eleven-species biofilm community, Chr was the dominant species across all the timepoints. In the reduced six-species biofilm community, however, Ent and 226 Pan became the most abundant species. These findings indicated that other species, although less 227 abundant or not associated with community members, also affect the final composition of the SynCom. 228 This result was consistent with a previous study that showed rare species were indispensable to the synergistic effects in multi-species biofilms (Ren et al., 2015). Furthermore, higher-order interactions only occur in the presence of additional species, emphasizing the importance of studying microbial interactions in the context of communities (Friedman et al., 2017).

232 Resource competition plays a major role in shaping bacterial communities, while metabolic exchanges 233 promote group survival (Goldford et al., 2018; Zelezniak et al., 2015). In the present study, we 234 simulated the metabolic interactions using metabolic models and assessed their predictability using 235 phenotypic assays. Specifically, communities with negative species exhibited higher resource 
236 competition, whereas communities with positive species displayed greater metabolite exchange.

237 These results indicate that metabolic interactions play a key role in determining the composition of

238 communities. Other mechanisms, such as niche partitioning created by spatial and temporal

239 heterogeneity, and trade-offs between nutrient acquisition and environmental tolerance, can also

240 promote coexistence between species (Louca et al., 2018). Our research provides insights into how

241 metabolic competition and cooperation simultaneously shape the community composition. The

242 methodology of network co-occurrence analysis combined with qPCR quantification, metabolic

243 modeling, and pair-wise interaction can be applied to various SynComs studies. Ultimately, such

244 studies should translate to a deeper understanding of how microbial communities behave in their

245 native environments, and this knowledge may be applied to wastewater treatment, disease

246 suppression, and crop yield enhancement.

\section{Materials and methods}

\section{Strains and genome sequencing}

249 As shown in Table 1, eleven bacterial isolates derived from the cucumber rhizosphere (Sun et al., 250 2021) were subjected to this study. These isolates were selected based on their co-existence in 251 biofilms (see Results).

Table 1. Strains used in this study

\begin{tabular}{ll}
\hline Strain & Abbreviation \\
\hline Achromobacter denitrificans XL100 & Ach \\
Acinetobacter baumannii XL380 & Aci \\
Bacillus velenzensis SQR9 & Bac \\
Burkholderia contaminans XL73 & Bur \\
Chryseobacterium rhizoplanae XL97 & Chr \\
Comamonas odontotermitis WLL & Com \\
Enterobacter bugandensis XL95 & Ent \\
Pantoea eucrina XL123 & Pan \\
\hline
\end{tabular}




\begin{tabular}{ll}
\hline Pseudomonas stutzeri XL272 & Pse \\
Pseudoxanthomonas japonensis XL7 & Pxa \\
Stenotrophomonas maltophilia XL133 & Ste \\
\hline
\end{tabular}

253 The whole genomes of the selected isolates were sequenced by different companies at different times.

254 The genomes of Aci, Com, Pxa and Ste were sequenced using a combination of PacBio RS II and 255 Illumina HiSeq 4000 sequencing platforms. The genome of Aci, Pxa and Ste were sequenced at the 256 Beijing Genomics Institute (BGI, Shenzhen, China). The Illumina data were used to evaluate the 257 complexity of the genome. Raw sequencing data and the assembled genome have been deposited 258 to the National Center for Biotechnology Information (NCBI) under the BioProject accession number 259 PRJNA593376, PRJNA762936 and PRJNA762715. The genome of Com was sequenced at Majorbio 260 Bio-Pharm Technology Co., Ltd. Raw sequencing data and the assembled genome have been 261 deposited to the NCBI under the BioProject accession number PRJNA762695.

262 The genomes of Bur, Chr, Ent, and Pan were sequenced using PacBio Sequel platform and Illumina 263 NovaSeq PE150 at the Beijing Novogene Bioinformatics Technology Co., Ltd. Raw sequencing data 264 and the assembled genomes have been deposited to the NCBI under the BioProject accession 265 number PRJNA593683, PRJNA721858, PRJNA761942, and PRJNA762676. Pse was sequenced by . 266 Genomes were automatically annotated by NCBI PGAP.

\section{Co-existence prediction}

268 To create a multi-species biofilm community, we adopted co-existence criteria. In parallel with our 269 previous study (Sun et al., 2021), rhizosphere soil of cucumber soil was collected. Two types of soil 270 were chosen: black soil and paddy soil. Bacterial abundance in the rhizosphere soil was roughly 271 estimated by CFU counting on TSB agar plates. Equal volumes of soil suspension were mixed with 272 B. velezensis SQR9 at an optical density at $600 \mathrm{~nm}\left(\mathrm{OD}_{600}\right)$ of 1 and inoculated in $10 \mathrm{ml}$ of TSB liquid 273 medium at a 1:100 ratio. Biofilms formed at the air-liquid interface and the solution underneath were 274 collected separately after $24 \mathrm{~h}$ of incubation at $30^{\circ} \mathrm{C}$. Each treatment had three biological replicates. 
275 Genomic DNA of the samples was extracted using an E.Z.N.A. Bacterial DNA Kit (Omega Bio-tek,

276 Inc.) following the manufacturer's instructions. Universal primers targeting the V3-V4 regions of the

277 16S rRNA gene were used to construct the DNA library for sequencing. Paired-end sequencing of

278 bacterial amplicons was performed on the Illumina MiSeq instrument (300 bp paired-end reads). Raw

279 sequencing data have been deposited to the NCBI SRA database under BioProject accession number

280 PRJNA739098. Reads were processed using the USEARCH pipeline. The paired-end reads were

281 merged using the "fastq_mergepairs" command. High-quality sequences were then selected using

282 the "fastq_filter" command and dereplicated using the "derep_fulllength" command. The singletons

283 were removed using "unoise3" algorithm and chimeric sequences were removed using "uchime_ref"

284 command. The remaining sequences were clustered to OTUs based on $97 \%$ sequence similarity. The

285 OTUs were classified at the genus and family level using the RDP database. The bacterial

286 composition in the biofilm was visualized in Microsoft Office Excel 2019 to predict co-existence.

\section{Initial 11-species SynCom growth condition}

288 The start inoculum was prepared by mixing equal volumes of species at an $\mathrm{OD}_{600}$ of 1 . The eleven289 species biofilm was cultivated by mixing $4 \mathrm{ml}$ of the mixed-species community (1\%) with $400 \mathrm{ml}$ of 290 tryptic soy broth (TSB) and incubating at $30^{\circ} \mathrm{C}$ for two to eight days.

292 Biofilms formed at the air-liquid interface were collected on days 2, 4, 6, and 8. Each time point had 293 eight biological replicates. Genomic DNA of the biofilm samples was extracted as previously 294 described. 16S rRNA gene amplicon sequencing was conducted and analyzed as described before. 295 The difference in the analysis is that the generated sequences were not clustered and directly used 296 to create ASV table. Taxonomy of the ASVs was assigned to the species with a reference database 297 consisting of the full 16S rRNA gene sequences of the eleven species. The compositional changes 298 were visualized in Microsoft Office Excel 2019. Microbial co-occurrence networks were constructed 299 to show the interactions among species during biofilm development. Spearman correlations among 300 all taxa were calculated using the R psych package. Only edges with correlation scores $>0.6$ were 
301 kept $(p<0.05$, FDR-adjusted). Correlation networks were visualized via Gephi using the Fruchterman

302 Reingold layout (Bastian et al., 2009).

\section{Reduced SynComs growth condition}

304 The four-, five- and six-species biofilms were grown in 6-well microtiter plates (VWR) insert with 100

$305 \mu \mathrm{m}$ sterile nylon mesh cell strainers (Biologix Cat \#15-1100). $10 \mathrm{ml}$ of TSB liquid medium and $100 \mu \mathrm{l}$

306 of start inoculum were added. The plates were incubated for $24 \mathrm{~h}$ at $30^{\circ} \mathrm{C}$ to allow the biofilm to grow

307 on top of the nylon mesh cell strainer.

\section{Reduced SynComs biomass quantification}

309 Biomass was defined by the fresh weight of biofilm. To measure the biomass, the cell strainer was

310 taken out, removed visible drops with paper, and weighed. The fresh weight was total weight minus

311 the weight of the nylon mesh. Each treatment had six biological replicates.

\section{Reduced SynComs cell numbers quantification by $\mathrm{QPCR}$}

313 To quantify the cell numbers of each isolate within the multi-species biofilm, strain-specific primers

314 were designed for the selected six isolates based on the whole-genome comparison. Genome 315 comparison was performed using Roary (Page et al., 2015). The primers target the single-copy gene 316 of the corresponding species. Conventional PCR and qPCR melt curves were used to evaluate the 317 specificity of the primers obtained. Primer sequences and target genes were listed in Table 2.

318 Standard curves were generated using plasmids containing corresponding fragments. qPCR was 319 performed with Applied Biosystems Real-Time PCR Instrument. Reaction components are as follow $3207.2 \mu \mathrm{l} \mathrm{H} \mathrm{H}_{2} \mathrm{O}, 10 \mu \mathrm{l} 2 \times$ ChamQ SYBR qPCR Master Mix (Vazyme), $0.4 \mu \mathrm{l} 10 \mu \mathrm{M}$ of each primer and 2 $321 \mu$ template DNA. The PCR programs were carried out under the following conditions: $95{ }^{\circ} \mathrm{C}$ for 10 $322 \mathrm{~min}, 40$ cycles of $95^{\circ} \mathrm{C}$ for $30 \mathrm{~s}, 60^{\circ} \mathrm{C}$ for $45 \mathrm{~s}$, followed by a standard melting curve segment. Each 323 treatment had six biological replicates, and each sample was run in triplicates (technical replicates).

Table 2. Primers used in this study 


\begin{tabular}{|c|c|c|c|}
\hline Primer & Sequence & $\begin{array}{l}\text { Product } \\
\text { size (bp) }\end{array}$ & $\begin{array}{l}\text { Target gene } \\
\text { locus_tag } \\
\text { (Genbank) }\end{array}$ \\
\hline Aci_F & ATTTAGTATCTGGTGAAGTCATCCGTA & 92 & GOD87_RS00290 \\
\hline Aci_R & CCGACAAATAAAGCTTGAGTAACTCC & & \\
\hline Com_F & CTCAAAACCAGTGTGATCGTGGAA & 109 & LAD35_RS00960 \\
\hline Com_R & TATTGCCCATCAGCAGAGTGTAGC & & \\
\hline Chr_F & GAACATCAGTTATCTTGTGAGCGGTA & 94 & KB553_RS00055 \\
\hline Chr_R & CATACAGGCTCCCATTCCTATTGTG & & \\
\hline Ent_F & AGCGTTACAGCAGCTACAGGATATTCACC & 96 & K9083_RS00400 \\
\hline Ent_R & СТTTTСАССАТСАССССАТСССТCGGTA & & \\
\hline Pan_F & TTAACATCGAAAAGCCTTCCCACCGTA & 101 & LAC65_RS01220 \\
\hline Pan_R & ATTCATCAGAAGCGCATGTATTACACT & & \\
\hline Pse_F & GAAATTCATCTTCGAACACAGCACAC & 124 & GOM96_RS02845 \\
\hline Pse_R & CTAGCTAACGGGGTTAAGTGCTTC & & \\
\hline
\end{tabular}

326 The direct competition of these isolates against each other was evaluated using the spot-on-lawn 327 assay and the pair-wise spot assay. Spot-on-lawn assay: $5 \mathrm{ml}$ of lawn species $\left(\mathrm{OD}_{600} \sim 0.02\right)$ grown 328 in TSB medium was spread onto a $25 \mathrm{ml}$ TSB plate (1.5\% agar) and removed by pipetting. Plates 329 were dried for 20 min. $5 \mu$ of spot species $\left(O_{600} \sim 0.4\right)$ grown in TSB medium was spot on the center 330 of the plates. Pair-wise spot assay: $5 \mu$ of the dual-species $\left(O_{600} \sim 1\right)$ grown in TSB medium were 331 spot on TSB plate (1.5\% agar) at $5 \mathrm{~mm}$ between the center of each colony. Plates were grown at $33230^{\circ} \mathrm{C}$ and imaged at $48 \mathrm{~h}$. The experiments were performed twice. 
334 The potential growth promotion of the bacterial metabolites to another species was evaluated using

335 a spent medium growth curve assay(Sun et al., 2021). Donor bacteria were grown in the M9 medium

336 with $1 \%$ glucose till the glucose was under detection. The cell culture was spun down, then the spent

337 medium was filter-sterilized and directly used as the medium of growth curve assay. $2 \mu$ l of recipient

338 species $\left(\mathrm{OD}_{600} \sim 1\right)$ was inoculated to $200 \mu \mathrm{l}$ spent medium or M9 glucose medium in a $10 \times 10$ well

339 Honeycomb Microplate. $\mathrm{OD}_{600}$ was measured every 30 minutes at $30{ }^{\circ} \mathrm{C}$ with Bioscreen $\mathrm{C}$ Automated

340 Microbiology Growth Curve Analysis System. Each treatment has 3 replicates. The experiments were

341 performed twice. The carrying capacity (maximum population size) was compared.

\section{Reduced SynComs genome-scale metabolic modeling}

343 Metabolic models were reconstructed using the CarveMe pipeline (Machado et al., 2018). The quality

344 of the metabolic models was validated using MEMOTE (Lieven et al., 2020). The metabolic interaction

345 potential and metabolic resource overlap for each community were analyzed using SMETANA

346 (Zelezniak et al., 2015; Zorrilla et al., 2021). The simulated cross-feeding results were summarized

347 as SMETANA score, which estimates the strength of metabolic exchanging (Zelezniak et al., 2015).

\section{Carbon source metabolic activity measurement}

349 We used PM1 (BIOLOG Cat \#13101) and PM2 (BIOLOG Cat \#13102) phenotypic microarrays to 350 assess the carbon source utilization ability of the community members (Bochner et al., 2001). The 351 assays were performed following the manufacturer's instructions. Briefly, $100 \mu$ of diluted cell 352 suspension of each species mixed with the BiOLOG redox dyes were added to each well of the PM 353 plates, and the plates inoculated with water and dyes were used as negative controls. All of the plates 354 were then incubated at $30{ }^{\circ} \mathrm{C}$ for up to $48 \mathrm{~h}$. If the species could utilize the carbon source in a well, 355 the colorless tetrazolium dye will be reduced to purple formazan by cell respiration. The color changes 356 were measured by an endpoint absorbance at $590 \mathrm{~nm}$ with a microplate reader. The variable level of 357 color changes indicates the carbon source metabolic activity. 
359 All the data needed to evaluate the conclusions in this paper are provided in the figures. Source data

360 related to this paper would be available online upon publication. Data were analyzed using R studio.

361 Statistical analysis methods were described in figure legends. A random forest approach was used 362 to identify the contribution of the six species responsible for the differences in community composition.

363 The analysis was performed using the $\mathrm{R}$ randomForest package (Andy Liaw et al., 2018). Plots were 364 generated using Microsoft Office Excel 2019 (stacked bar plot), Graphpad prism 8 (heatmap), R 365 ggplot2, ggpubr, ggalluvial, pheatmap packages, and Adobe Illustrator CC 2020 (Adobe Inc.). 366 Schematic diagrams were generated using BioRender.

\section{Acknowledgments}

368 This work was financially supported by the National Nature Science Foundation of China (31972512 369 and 42090064), the Innovative Research Team Development Plan of the Ministry of Education of 370 China (Grant No. IRT_17R56), the Fundamental Research Funds for the Central Universities 371 (KYXK202009). XS was supported by a Chinese Scholarship Council fellowship. ÁTK was supported

372 by the Danish National Research Foundation (DNRF137) for the Center for Microbial Secondary 373 Metabolites and the Novo Nordisk Foundation via the INTERACT project (grant number 374 NNF19SA0059360). During her unexpected trap in Denmark caused by the pandemic of Omicron, 375 author XS is extremely grateful to ZX for his financial support, and to Danish guitarist Niklas Johansen 376 for his weekly lessons and constant spiritual support.

\section{Competing Interests}

378 The authors declare that there are no competing financial interests related to the work described.

\section{Author contributions}

380 XS, JX, ZX, ÁTK designed the study, XS, JX, RX performed the experiments. DZ and WW performed the metabolic modeling. XS and JX analyzed the data and created the figures. XS wrote the first draft of the manuscript, ZX, ÁTK, QS revised the manuscript. 
384 Abram F. 2015. Systems-based approaches to unravel multi-species microbial community functioning. Computational and Structural Biotechnology Journal 13:24-32. doi:10.1016/J.CSBJ.2014.11.009

Barberán A, Bates ST, Casamayor EO, Fierer N. 2012. Using network analysis to explore cooccurrence patterns in soil microbial communities. ISME Journal 6:343-351. doi:10.1038/ismej.2011.119

Blasche S, Kim Y, Oliveira AP, Patil KR. 2017. Model microbial communities for ecosystems biology.

398 Breugelmans P, Barken KB, Tolker-Nielsen T, Hofkens J, Dejonghe W, Springael D. 2008. Architecture and spatial organization in a triple-species bacterial biofilm synergistically

402 Cavaliere M, Feng S, Soyer OS, Jiménez JI. 2017. Cooperation in microbial communities and their biotechnological applications. Environmental Microbiology 19:2949-2963. doi:10.1111/1462- 
405 Chan SHJ, Simons MN, Maranas CD. 2017. SteadyCom: Predicting microbial abundances while ensuring community stability. PLOS Computational Biology 13:e1005539. doi:10.1371/JOURNAL.PCBI.1005539

Cho I, Blaser MJ. 2012. The human microbiome: at the interface of health and disease. Nature Reviews Genetics 2012 13:4 13:260-270. doi:10.1038/nrg3182

410 Durán P, Thiergart T, Garrido-Oter R, Agler M, Kemen E, Schulze-Lefert P, Hacquard S. 2018. 983.e14. doi:10.1016/j.cell.2018.10.020

413 Estrela S, Sanchez-Gorostiaga A, Vila JCC, Sanchez A. 2021. Nutrient dominance governs the assembly of microbial communities in mixed nutrient environments. eLife 10.

416 Faust K. 2021. Open challenges for microbial network construction and analysis. The ISME Journal 1-8. doi:10.1038/s41396-021-01027-4

418 Faust K, Sathirapongsasuti JF, Izard J, Segata N, Gevers D, Raes J, Huttenhower C. 2012. Microbial co-occurrence relationships in the human microbiome. PLoS Computational Biology 8.

421 Fitzpatrick CR, Salas-González I, Conway JM, Finkel OM, Gilbert S, Russ D, Teixeira PJPL, DangI JL. doi:10.1371/journal.pcbi.1002606

424 Foster KR, Bell T. 2012. Competition, not cooperation, dominates interactions among culturable microbial species. Current Biology 22:1845-1850. doi:10.1016/j.cub.2012.08.005 
426 Friedman J, Higgins LM, Gore J. 2017. Community structure follows simple assembly rules in microbial microcosms. Nature Ecology and Evolution 1:1-7. doi:10.1038/s41559-017-0109

428 Fu H, Uchimiya M, Gore J, Moran MA. 2020. Ecological drivers of bacterial community assembly in synthetic phycospheres. Proceedings of the National Academy of Sciences of the United States

Gao C-H, Cao H, Cai P, Sørensen SJ. 2020. The initial inoculation ratio regulates bacterial coculture interactions and metabolic capacity. The ISME Journal 1-12. doi:10.1038/s41396-020-00751-7

Gao CH, Zhang M, Wu Y, Huang Q, Cai P. 2019. Divergent Influence to a Pathogen Invader by Resident Bacteria with Different Social Interactions. Microbial Ecology 77:76-86.

Goldford JE, Lu N, Bajić D, Estrela S, Tikhonov M, Sanchez-Gorostiaga A, Segrè D, Mehta P, Sanchez doi:10.1007/s00248-018-1207-z

Heinken A, Basile A, Hertel J, Thinnes C, Thiele I. 2021. Genome-scale metabolic modeling of the doi:10.1126/science.aat1168

Gómez-Godínez L, Martínez-Romero E, Trejo JB, Arteaga-Garibay RI. 2021. Tools and challenges to exploit microbial communities in agriculture. Current Research in Microbial Sciences 2:100062. doi:10.1016/j.crmicr.2021.100062 
448 Lee K, Yoon SS. 2017. <i>Pseudomonas aeruginosa<i> biofilm, a programmed bacterial life for fitness. Journal of Microbiology and Biotechnology 27:1053-1064. doi:10.4014/jmb.1611.11056

Lee KWK, Periasamy S, Mukherjee M, Xie C, Kjelleberg S, Rice SA. 2014. Biofilm development and enhanced stress resistance of a model, mixed-species community biofilm. ISME Journal 8:894907. doi:10.1038/ismej.2013.194 Malik Sheriff RS, Shoaie S, Sonnenschein N, Teusink B, Vilaça P, Vik JO, Wodke JAH, Xavier JC, Yuan Q, Zakhartsev M, Zhang C. 2020. MEMOTE for standardized genome-scale metabolic model testing. Nature Biotechnology 2020 38:3 38:272-276. doi:10.1038/s41587-020-0446-y

Liu W, Jacquiod S, Brejnrod A, Russel J, Burmølle M, Sørensen SJ. 2019. Deciphering links between bacterial interactions and spatial organization in multispecies biofilms. ISME Journal 13:30543066. doi:10.1038/s41396-019-0494-9 microbial systems. Nature Ecology \& Evolution 2018 2:6 2:936-943. doi:10.1038/s41559-0180519-1 
471 Machado D, Andrejev S, Tramontano M, Patil KR. 2018. Fast automated reconstruction of genomescale metabolic models for microbial species and communities. Nucleic Acids Research 46:7542-7553. doi:10.1093/NAR/GKY537

474 Nielsen AT, Tolker-Nielsen T, Barken KB, Molin S. 2000. Role of commensal relationships on the spatial structure of a surface-attached microbial consortium. Environmental Microbiology

477 Niu B, Paulson JN, Zheng X, Kolter R. 2017. Simplified and representative bacterial community of maize roots. Proceedings of the National Academy of Sciences of the United States of America 480 Oliveira NM, Martinez-Garcia E, Xavier J, Durham WM, Kolter R, Kim W, Foster KR. 2015. Biofilm 114:E2450-E2459. doi:10.1073/pnas.1616148114

Ortiz A, Vega NM, Ratzke C, Gore J. 2021. Interspecies bacterial competition regulates community assembly in the C. elegans intestine. ISME Journal 1-12. doi:10.1038/s41396-021-00910-4 assemblages for disease management. Phytopathology 106:1083-1096. doi:10.1094/PHYTO- 
492 Raghupathi PK, Liu W, Sabbe K, Houf K, Burmølle M, Sørensen SJ. 2018. Synergistic Interactions within a Multispecies Biofilm Enhance Individual Species Protection against Grazing by a Pelagic Protozoan. Frontiers in Microbiology 8:2649. doi:10.3389/fmicb.2017.02649

Ratzke C, Barrere J, Gore J. 2020. Strength of species interactions determines biodiversity and stability in microbial communities. Nature Ecology and Evolution 4:376-383.

Ren D, Madsen JS, Sørensen SJ, Burmølle M. 2015. High prevalence of biofilm synergy among

501 Rendueles O, Ghigo JM. 2012. Multi-species biofilms: how to avoid unfriendly neighbors. FEMS Microbiology Reviews 36:972-989. doi:10.1111/j.1574-6976.2012.00328.x

Sadiq FA, Burmølle M, Heyndrickx M, Flint S, Lu W, Chen W, Zhao J, Zhang H. 2021. Communitywide changes reflecting bacterial interspecific interactions in multispecies biofilms. Critical

Sun X, Xu Z, Xie J, Hesselberg-Thomsen V, Tan T, Zheng D, Strube ML, Dragoš A, Shen Q, Zhang R, Reviews in Microbiology 1-21. doi:10.1080/1040841x.2021.1887079 Kovács ÁT. 2021. Bacillus velezensis stimulates resident rhizosphere Pseudomonas stutzeri for plant health through metabolic interactions. ISME Journal 1-14. doi:10.1038/s41396-02101125-3

Wei Z, Gu Y, Friman VP, Kowalchuk GA, Xu Y, Shen Q, Jousset A. 2019. Initial soil microbiome composition and functioning predetermine future plant health. Science Advances 5:759-784. doi:10.1126/sciadv.aaw0759 
513 Weiss AS, Burrichter AG, Chakravarthy A, Raj D, von Strempel A, Meng C, Kleigrewe K, Münch PC,

Rössler L, Huber C, Eisenreich W, Jochum LM, Göing S, Jung K, Lincetto C, Hübner J, Marinos G, Zimmermann J, Kaleta C, Sanchez A, Stecher B. 2021. In vitro interaction network of a synthetic gut bacterial community. The ISME Journal 2021 1-15. doi:10.1038/s41396-021-01153-z

517 Widder S, Allen RJ, Pfeiffer T, Curtis TP, Wiuf C, Sloan WT, Cordero OX, Brown SP, Momeni B, Shou W, Kettle H, Flint HJ, Haas AF, Laroche B, Kreft J-U, Rainey PB, Freilich S, Schuster S, Milferstedt K, van der Meer JR, Großkopf T, Huisman J, Free A, Picioreanu C, Quince C, Klapper I, Labarthe S, Smets BF, Wang H, Soyer OS. 2016. Challenges in microbial ecology: building predictive understanding of community function and dynamics. The ISME Journal 2016 10:11 10:25572568. doi:10.1038/ismej.2016.45

523 Zelezniak A, Andrejev S, Ponomarova O, Mende DR, Bork P, Patil KR. 2015. Metabolic dependencies drive species co-occurrence in diverse microbial communities. Proceedings of the National Academy of Sciences 112:6449-6454. doi:10.1073/PNAS.1421834112

526 Zorrilla F, Buric F, Patil KR, Zelezniak A. 2021. metaGEM: reconstruction of genome scale metabolic models directly from metagenomes. Nucleic Acids Research 49:e126-e126. 